\title{
Screening and clinical evaluation of dominant peptides of centromere protein $F$ antigen for early diagnosis of hepatocellular carcinoma
}

\author{
SIWEN LI ${ }^{1}$, XIAOJIN LI ${ }^{1}$, ANJIAN XU ${ }^{1}$, BEI ZHANG $^{1}$, XIAOMIN HE ${ }^{1}$, HONGDA CHEN $^{2}$ and JIAN HUANG ${ }^{1,3,4}$ \\ ${ }^{1}$ Liver Research Center, Experimental Center, Beijing Friendship Hospital, Capital Medical University, \\ Beijing Key Laboratory of Translational Medicine in Liver Cirrhosis, Beijing, 100050; ${ }^{2}$ Program Office \\ for Cancer Screening in Urban China, National Cancer Center/Cancer Hospital, Chinese Academy of \\ Medical Sciences and Peking Union Medical College, Beijing 100021; ${ }^{3}$ National Clinical Research Center \\ of Digestive Diseases, Beijing Friendship Hospital, Capital Medical University; ${ }^{4}$ Beijing Key Laboratory \\ of Tolerance Induction and Organ Protection in Transplantation, Beijing 100050, P.R. China
}

Received June 20, 2017; Accepted November 22, 2017

DOI: $10.3892 / \mathrm{mmr} .2018 .8372$

\begin{abstract}
Tumor-associated antigens, such as centromere protein F (CENP-F), have been recognized as potential serological biomarkers for early diagnosis of hepatocellular carcinoma (HCC); however, the exact regions corresponding to the dominant peptides of CENP-F antigen remain to be explored. We aimed to screen and evaluate potential dominant peptides of CENP-F for early diagnosis of HCC. Dominant peptides of CENP-F were predicted by BioSun version 3.0, and the corresponding recombinant proteins were prepared. Enzyme-linked immunosorbent assays were conducted for initial screening of dominant peptides, and selected dominant peptides were subjected to further clinical evaluation. Eight dominant peptides of CENP-F antigens were predicted at amino acids (a.a) 121-220, 335-416, 1100-1265, 1670-1791, 1759-2093, 2075-2210, 2485-2592, and 2808-2960. Initial screening of the predicted peptides in samples of $47 \mathrm{HCC}$ cases showed the highest diagnostic value for 121-220 a.a and 1670-1791 a.a peptides with area under the curve (AUC) values of 0.795 [95\% confidence interval (CI), 0.706-0.884] and 0.809 (95\% CI, 0.721-0.896),
\end{abstract}

Correspondence to: Dr Jian Huang, Liver Research Center, Experimental Center, Beijing Friendship Hospital, Capital Medical University, Beijing Key Laboratory of Translational Medicine in Liver Cirrhosis, 95 Yongan Street, Beijing 100050, P.R. China E-mail: huangj1966@hotmail.com

Dr Hongda Chen, Program Office for Cancer Screening in Urban China, National Cancer Center/Cancer Hospital, Chinese Academy of Medical Sciences and Peking Union Medical College, 17 Panjiayuannanli, Beijing 100021, P.R. China

E-mail: hongda.chen@cicams.ac.cn

Key words: centromere protein F, dominant peptide, autoantibody, hepatocellular carcinoma, early diagnosis sensitivity of 58.3 and $85.4 \%$, and specificity of 93.9 and $65.3 \%$, respectively. Further evaluation of the two peptides in 405 samples comprised of $153 \mathrm{HCC}, 126$ liver cirrhosis and 126 healthy controls, presenting an AUC of 0.743 (95\% CI, 0.674-0.812) for 121-220 a.a peptide in detecting early-stage HCCs. Specifically, the 121-220 a.a peptide showed a complementary effect in combination with $\alpha$-fetoprotein (AFP) for the detection of early-stage HCC with increased AUC value of 0.840 (95\% CI, 0.781-0.899), and sensitivity of $81.4 \%$ and specificity of $72.2 \%$. In conclusion, our study identified the 121-220 a.a dominant peptide as the region of CENP-F antigen with the highest immunogenicity and demonstrated its value in combination with AFP for diagnosis of early-stage HCC.

\section{Introduction}

Hepatocellular carcinoma (HCC) is the sixth most common malignant disease and the third leading cause of cancer-related death worldwide (1). Most patients with HCC are diagnosed at a later stage of disease, leading to poor prognosis with a 5-year survival rate of less than $16 \%$ (2). However, the 5-year survival rate increases to more than $50 \%$ for HCC detected at early stages (2). Therefore, searching for biomarkers for detecting early-stage $\mathrm{HCC}$ is critical in improvement of the overall prognosis of HCC.

At present, imaging techniques and determination of $\alpha$-fetoprotein (AFP) levels are widely used as screening tools. Because of the high cost and radiation exposure, or insufficient sensitivity and specificity, imaging techniques have limitations in the screening of early $\mathrm{HCC}(3,4)$. AFP is currently the main serum biomarker used in the diagnosis of HCC, with low sensitivity of $46-59 \%$ and specificity of $87-93 \%$ for detection of early HCC (5). Other biomarkers reported in recent years include lens culinaris agglutinin-reactive fraction of AFP (AFP-L3), des- $\gamma$-carboxyprothrombin (DCP), squamous cell carcinoma antigen (SCCA), and golgi protein 73 (GP73) (6-10), with area under the curves (AUCs) of 0.67 to 0.77 , sensitivities 
of $28.6-70.9 \%$, and specificities of $74.9-92.7 \%$ for early-stage $\mathrm{HCC}(9-13)$.

In recent years, tumor-associated antigens (TAAs) with promising diagnostic value for tumors at early stages, including HCC, have been identified (14). Among them, serological autoantibody to centromere protein F (CENP-F) has been recognized to have potential value in the detection of early HCC $(14,15)$. CENP-F is a kinetochore protein of 3,210 amino acids that plays a role in centromere formation and kinetochore organization during mitosis (16-21). Using protein microarray to evaluate the diagnostic value of CENP-F autoantibody in a large HCC cohort, our previous study showed that CENP-F antibody had better sensitivity for the detection of early-stage HCC compared with AFP and that combined autoantibody to CENP-F with AFP further improved the diagnostic capability for early HCC (22). However, much less has been known regarding the dominant epitopes of CENP-F antigen, and the exact regions corresponding to the dominant peptides of CENP-F antigen remain to be explored.

In the present study, we aimed to screen and evaluate potential dominant epitope peptides in the full-length CENP-F antigen protein with the aim of obtaining novel CENP-F antigens and improving the early diagnosis of HCC.

\section{Materials and methods}

Study population. Screening group: for screening of antigens with the best serodiagnostic performance among eight individual antigens we collected serum samples including 47 cases of HCC (38 men and 9 women aged 44-80 years, with a median age of 57.0 years) and 48 healthy controls (21 men and 27 women aged 20-59 years, with a median age of 46.0 years). Validation group: For validation of the serodiagnostic performance of the selected antigen dominant epitopes we collected another set of 405 serum samples, including 153 cases of HCC with AFP data available (127 men and 26 women aged 0-81 years, with a median age of 57.0 years), of which 70 cases were early-stage HCC (57 men and 13 women aged $28-80$ years, with a median age of 56.5 years); 126 cases of liver cirrhosis ( 95 men and 31 women aged 27-73 years, with a median age of 51.0 years); and 126 healthy controls (65 men and 61 women aged 20-70 years, with a median age of 47.0 years). Clinical characteristics of the samples are shown in Table I.

All samples were obtained from the Cancer Hospital, Chinese Academy of Medical Science, Beijing, China; Beijing Youan Hospital, Capital Medical University, Beijing, China; and Beijing Friendship Hospital, Capital Medical University (Beijing, China) from November 2013 to December 2016.

A diagnosis of HCC was based on the guideline for diagnosis and treatment of primary HCC (2012 version, China). Early-stage HCC was defined as a tumor at TMN stage I. Diagnosis of LC was based on ultrasound, computed tomography (CT), or magnetic resonance imaging (MRI) characteristics, laboratory indexes, and histopathology (3). Healthy controls were healthy examiners with normal liver biochemistry, no history of liver disease, and no malignant disease.

All serum samples were stored at $-80^{\circ} \mathrm{C}$ until testing. The study protocol was approved by the Clinical Research Ethics
Committee of Beijing Friendship Hospital, Capital Medical University (Beijing, China).

Bioinformatics analysis of dominant epitope peptides of $C E N P-F$. Candidate dominant epitopes of CENP-F protein were predicted using BioSun version 3.0 software developed by the Center of Computational Biology, Beijing Institute of Basic Medical Sciences (Beijing, China). Based on the epitope curve, peptides containing the dominant CENP-F epitopes with highest peak values were selected as the target peptides.

Construction, expression, and purification of recombinant proteins. Coding sequences of each dominant antigen peptide of eight single antigens (121-220 a.a, 335-416 a.a, 1100-1265 a.a, 1670-1791 a.a, 1759-2093 a.a, 2075-2210 a.a, 2485-2592 a.a, 2808-2960 a.a) with GST or His tags were chemically synthesized and inserted into the prokaryotic expression plasmid pET-6P with GST or 6-His tags (constructed in house) using specific endonuclease restriction sites BamHI and XhoI. The recombinant plasmids were transformed into Escherichia coli BL21 or BL21 (DE3) and the fusion proteins were expressed following induction with $0.1 \mathrm{M}$ isopropyl $\beta$-D-thiogalactoside at $16^{\circ} \mathrm{C}$ for $12 \mathrm{~h}$. The soluble expression of recombinant proteins were purified by affinity chromatography using GST-Sefinose resin or His-Sefinose resin (Sangon Biotech Co., Ltd., Shanghai, China). The purity of fusion proteins was analyzed by sodium alt-polyacrylamide gel electrophoresis (SDS-PAGE) and Gel-Pro Analyzer version 3.1.00.00 (Media Cybernetics, Inc., Silver Spring, MD, USA) and the protein concentration was determined by the bicinchoninic acid (BCA) method (Pierce, Rockford, IL, USA).

Enzyme-linked immunosorbent assay (ELISA) for screening and evaluation of dominant peptides. For ELISA, 96-well microplates (Nunc A/S, Roskilde, Denmark) were coated with individual antigens at $5 \mu \mathrm{g} / \mathrm{ml}(100 \mu \mathrm{l} /$ well $)$ in coating buffer (0.05 M carbonate/bicarbonate, $\mathrm{pH} 9.6$ ) and incubated at $4{ }^{\circ} \mathrm{C}$ overnight. The plates were washed once with phosphate-buffered saline (PBS) containing $0.05 \%$ Tween-20 and blocked by the addition of $200 \mu \mathrm{l}$ of $10 \%$ newborn bovine serum (Life Technologies, Burlington, ON, Canada) and incubation at $37^{\circ} \mathrm{C}$ for $2 \mathrm{~h}$. Next, $100 \mu \mathrm{l}$ of standard serum (in-house preparation) diluted 1:4 $(1,000 \mu \mathrm{g} / \mathrm{ml}), 1: 8(500 \mu \mathrm{g} / \mathrm{ml}), 1: 16(250 \mu \mathrm{g} / \mathrm{ml})$, 1:32 (125 $\mu \mathrm{g} / \mathrm{ml}), 1: 64(62.5 \mu \mathrm{g} / \mathrm{ml}), 1: 128(31.25 \mu \mathrm{g} / \mathrm{ml}), 1: 256$ $(15.625 \mu \mathrm{g} / \mathrm{ml})$, and $1: 512(7.8125 \mu \mathrm{g} / \mathrm{ml})$ in $10 \%$ newborn bovine serum or $100 \mu \mathrm{l}$ of patient serum diluted 1:11 in PBS containing $10 \%$ newborn bovine serum was added to the wells and incubated for $1 \mathrm{~h}$ at $37^{\circ} \mathrm{C}$. The plates were washed five times and then $100 \mu \mathrm{l}$ of a 1:8,000 dilution of rabbit anti-human IgG-peroxidase antibody (Sigma-Aldrich, St. Louis, MO, USA) was added and incubated for $30 \mathrm{~min}$ at $37^{\circ} \mathrm{C}$, followed by addition of $100 \mu 1$ TMB HRP-Substrate (Beijing Solarbio Science \& Technology Co., Ltd., Beijing, China) and incubation for $10 \mathrm{~min}$ at $37^{\circ} \mathrm{C}$. The reaction was stopped by addition of $50 \mu 1$ stop solution (Beijing Solarbio Science \& Technology Co., Ltd.) and absorbance was immediately read at $450 \mathrm{~nm}$ or $630 \mathrm{~nm}$ using a microplate reader SpectraMax M3 (Molecular Devices, LLC, Sunnyvale, CA, USA). 
Table I. Study population characteristics.

\begin{tabular}{|c|c|c|c|}
\hline Characteristics & $\mathrm{HCC}(\mathrm{N}=153)$ & $\mathrm{LC}(\mathrm{N}=126)$ & $\mathrm{HC}(\mathrm{N}=126)$ \\
\hline Age $($ mean $\pm \mathrm{SD})$ & $56.8 \pm 11.2$ & $50.0 \pm 9.7$ & $46.9 \pm 8.5$ \\
\hline \multicolumn{4}{|l|}{$\operatorname{Sex}(n, \%)$} \\
\hline Male & $127(83.0)$ & $95(75.4)$ & $65(51.6)$ \\
\hline Female & $26(17.0)$ & $31(24.6)$ & $61(48.4)$ \\
\hline \multicolumn{4}{|c|}{ HBV infection (n, \%) } \\
\hline $\mathrm{HBV}(+)$ & $107(69.9)$ & $101(80.2)$ & $0(0.0)$ \\
\hline HBV (-) & $44(28.8)$ & $25(19.8)$ & $126(100.0)$ \\
\hline Missing & $2(1.3)$ & $0(0.0)$ & $0(0.0)$ \\
\hline \multicolumn{4}{|c|}{ HCV infection (n, \%) } \\
\hline $\mathrm{HCV}(+)$ & $13(8.5)$ & $0(0.0)$ & $0(0.0)$ \\
\hline $\mathrm{HCV}(-)$ & $138(90.2)$ & $126(100.0)$ & $126(100.0)$ \\
\hline Missing & $2(1.3)$ & $0(0.0)$ & $0(0.0)$ \\
\hline \multicolumn{4}{|c|}{ TNM tumor stage $(\mathrm{n}, \%)$} \\
\hline I & $70(45.8)$ & - & - \\
\hline$>\mathrm{I}$ & $83(54.2)$ & - & - \\
\hline \multicolumn{4}{|l|}{ Child-Pugh (n, \%) } \\
\hline A & $83(54.2)$ & $57(45.2)$ & - \\
\hline $\mathrm{B}$ & $23(15.0)$ & $33(26.2)$ & - \\
\hline $\mathrm{C}$ & $14(9.2)$ & $23(18.3)$ & - \\
\hline Missing & 33 (21.6) & $13(10.3)$ & - \\
\hline \multicolumn{4}{|l|}{ AFP } \\
\hline$\geq 20 \mathrm{ng} / \mathrm{ml}$ & $81(52.9)$ & $22(17.5)$ & $0(0.0)$ \\
\hline$<20 \mathrm{ng} / \mathrm{ml}$ & $72(47.1)$ & $104(82.5)$ & $126(100.0)$ \\
\hline AST (U/l) & $54.2(11.0-659.5)$ & $35.2(13.9-393.7)$ & - \\
\hline ALT (U/l) & $43.3(8.2-837.4)$ & $30.6(8.2-1328.0)$ & - \\
\hline ALB (g/l) & $35.9(22.1-63.4)$ & $36.2(3.9-49.4)$ & - \\
\hline TBIL $(\mu \mathrm{mol} / \mathrm{l})$ & $32.5(6.9-945.0)$ & $23.9(5.1-475.1)$ & - \\
\hline $\mathrm{DBIL}(\mu \mathrm{mol} / \mathrm{l})$ & $6.3(0.9-243.0)$ & $5.6(1.0-221.7)$ & - \\
\hline
\end{tabular}

Continuous variables are expressed as the mean \pm SD or the medians. Count data are described as frequency. HCC, hepatocellular carcinoma; LC, liver cirrhosis; HC, healthy controls; SD, standard deviation; HBV, hepatitis B virus; HCV, hepatitis C virus; TNM, tumor node metastasis; AFP, $\alpha$-fetoprotein; AST, aspartate transaminase; ALT, alanine aminotransferase; ALB, albumin; TBIL, total bilirubin; DBIL, direct bilirubin .

Statistical analysis. All statistical analyses were performed using SPSS (version 23.0; IBM Corp., Armonk, NY, USA) and GraphPad Prism (version 6.0c; GraphPad Software, Inc., La Jolla, CA, USA). Receiver operating characteristic (ROC) curves were plotted and the following diagnosis-related indicators, including sensitivity, specificity and AUCs along with $95 \%$ confidence intervals $[95 \%$ confidence intervals (CIs)], were used to evaluate the diagnostic performance of individual biomarkers. The respective optimal cut-off values of individual biomarkers in detecting HCCs were determined by the Youden's index (sensitivity + specificity-1). We used the Chi-square $\left(\chi^{2}\right)$ test to evaluate the correlation between the level of auto-antibody to CENP-F and TNM stage of HCC.

In addition, we further evaluated the diagnostic potential of multiple biomarkers using logistic regression models. The predicted probabilities were used to conduct ROC analyses, and diagnosis-related indicators were therefore calculated and reported.

\section{Results}

Prediction of peptides containing dominant epitopes of CENP-F. Based on the epitope curve (Fig. 1A), eight peptides containing CENP-F dominant epitopes with higher peak values for each antigen were determined as follows: 121-220 a.a peptide, 335-416 a a peptide, $1100-1265$ a.a peptide, 1670-1791 a.a peptide, 1759-2093 a.a peptide, 2075-2210 a.a peptide, 2485-2592 a.a peptide, and 2808-2960 a.a peptide. The eight peptides covered all functional domains except for the domain responsible for 2x96 AA approximate tandem repeats (Fig. 1B). Recombinant proteins of the eight predicted dominant peptides were prepared, including 1759-2093 a.a peptide with his tag, and other seven peptides with GST tag (Fig. 2).

Serological reactivity of the eight individual antigen peptides. We used an indirect ELISA to evaluate the diagnostic 


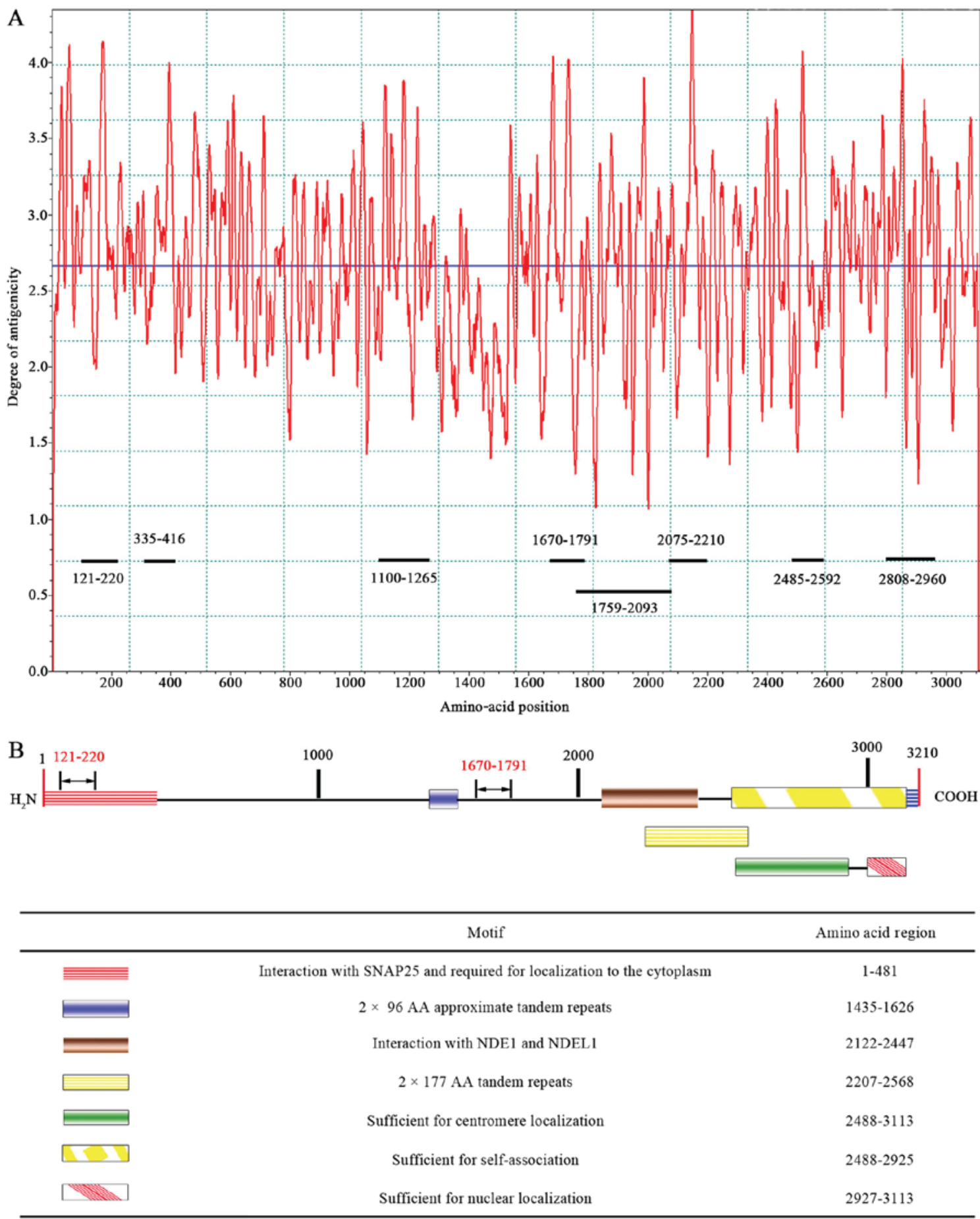

Figure 1. (A) Epitope curves for the eight predicted dominant peptides, and (B) schematic presentation of CENP-F domains and sequence motifs. CENP-F, centromere protein F.

performance of the individual peptides by the analysis of anti-CENP-F level in serum of 47 cases of HCC and 48 healthy control. The AUC value and sensitivities and specificities of the eight individual antigen peptides are shown in Table II. The scatter plots showing the eight antigens of CENP-F between $\mathrm{HCC}$ and $\mathrm{HC}$ groups are presented in Fig. 3, and the ROC curves of the eight individual CENP-F antigens in discriminating between HCC and healthy controls (HC) are shown in Fig. 4. The sensitivities of the eight individual peptide antigens ranged from 25.50 to $85.40 \%$, and the specificities ranged from 59.20 to $93.30 \%$. Among them, two peptides of CENP-F, 121-220 a.a and 1670-1791 a.a, had the highest diagnostic value for HCC with AUC values of 0.795 and 0.809 , respectively. Specificity was better for peptide 121-220 a.a (93.9\%) but sensitivity was better for peptide 1670-1791 a.a (Figs. 3 and 4, Table II).

Validation of the diagnostic performance of the 121-220 a.a and 1670-1791 a.a antigen peptides. A total of 405 serum samples from HCC, LC, and HC were used to evaluate the 
Table II. Diagnostic performance of the eight predicted dominant peptides of CENP-F antigen evaluated by ELISA analysis of samples of screening group.

\begin{tabular}{|c|c|c|c|c|c|}
\hline Dominant peptides & AUC value & $95 \% \mathrm{CI}$ & Sensitivity (\%) & Specificity (\%) & Cut-off value \\
\hline $121-220$ a.a & 0.795 & $0.706-0.884$ & 58.3 & 93.9 & 154 \\
\hline 335-416 a.a & 0.711 & $0.606-0.815$ & 54.2 & 87.8 & 225 \\
\hline $1100-1265$ a.a & 0.614 & $0.501-0.727$ & 60.4 & 63.3 & 250 \\
\hline 1670-1791 a.a & 0.809 & $0.721-0.896$ & 85.4 & 65.3 & 458 \\
\hline 1759-2093 a.a & 0.513 & $0.397-0.629$ & 25.0 & 85.7 & 793 \\
\hline 2075-2210 a.a & 0.630 & $0.520-0.740$ & 22.9 & 98.0 & 441 \\
\hline 2485-2592 a.a & 0.682 & $0.576-0.788$ & 68.8 & 59.2 & 276 \\
\hline 2808-2960 a.a & 0.656 & $0.547-0.766$ & 72.9 & 59.2 & 163 \\
\hline
\end{tabular}

a.a, amino acid; AUC, area under curve; CI, confidence interval; CENP-F, centromere protein F; ELISA, enzyme-linked immunosorbent assay.

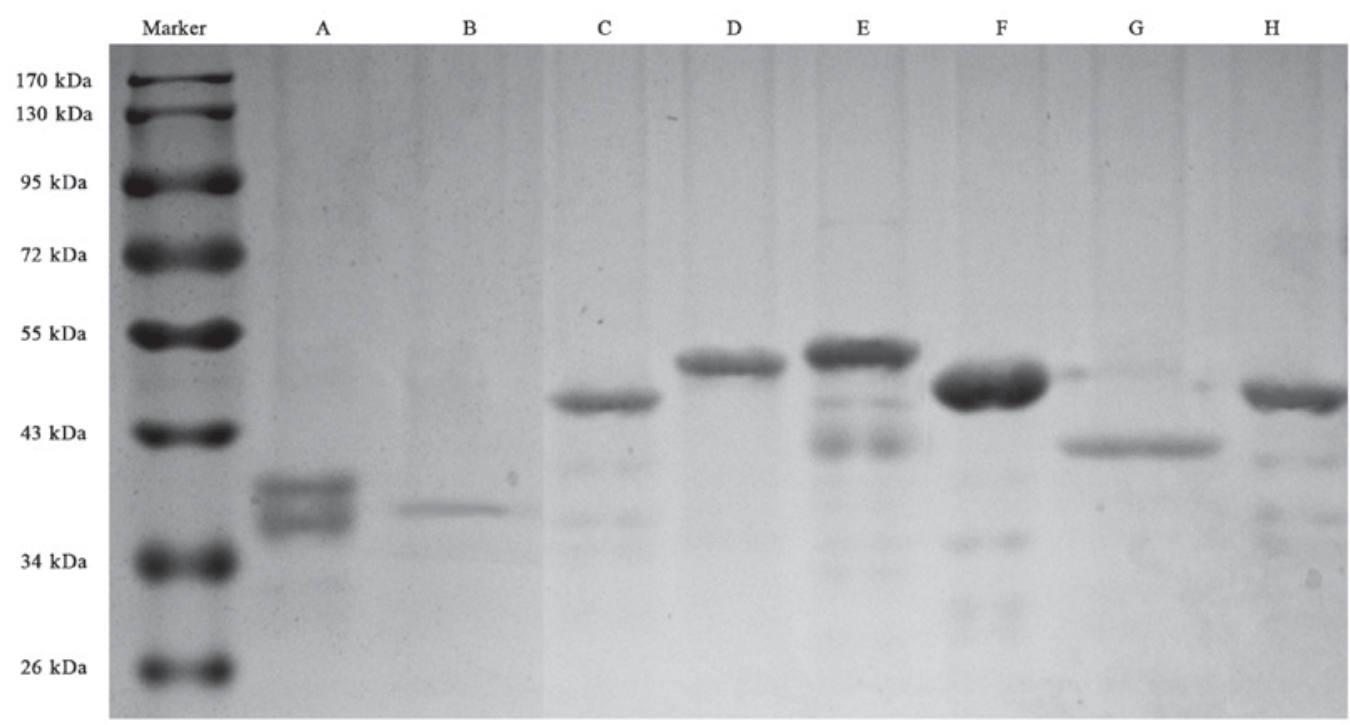

Figure 2. SDS-PAGE electrophoresis for the eight recombinant fragments of CENP-F protein. (A), 121-220 a.a with GST tag, with a degraded band (with CENP-F antigenicity); (B), 335-416 a.a with GST tag; (C), 1759-2093 a.a with his tag; (D), 1100-1265 a.a with GST tag; E, 1670-1791 a.a with GST tag; F, 2075-2210 a.a with GST tag; G, 2485-2592 a.a with GST tag; H, 2808-2960 a.a with GST tag. SDS-PAGE, sodium alt-polyacrylamide gel electrophoresis; CENP-F, centromere protein F; a.a, amino acid.

diagnostic value of the two antigen peptides 121-220 a.a and 1670-1791 a.a, as well as the effect of combined antigen peptide with AFP, for the detection of HCC.

As shown in Table III, the 121-220 a.a peptide gave results consistent with the data obtained in the screening group; the AUC value for the discrimination of $\mathrm{HCC}$ from $\mathrm{HC}$ was 0.749 (95\% CI, 0.692-0.807) with sensitivity of $68 \%$ and specificity of $72.2 \%$, and the AUC for discrimination of early HCC from $\mathrm{HC}$ was 0.743 (95\% CI, 0.674-0.812) with sensitivity of 68.6 and specificity of $72.2 \%$, but there was no significant difference between HCC or early HCC and LC. However, the 1670-1791 a.a peptide showed lower performance compared with the results in the screening group; the AUC value for the discrimination of $\mathrm{HCC}$ from $\mathrm{HC}$ was 0.628 (95\% CI, 0.563-0.693) with sensitivity of $62.1 \%$ and specificity of $61.9 \%$, and the AUC for discrimination of early $\mathrm{HCC}$ from $\mathrm{HC}$ was 0.584 (95\% CI, 0.500-0.668) with sensitivity of $72.9 \%$ and specificity of $50.0 \%$, with no significant difference between HCC or early HCC and LC.
Specifically, the combination of AFP, for which a serum level greater than $20 \mathrm{ng} / \mathrm{ml}$ was defined as positive, and autoantibody to 121-220 a.a dominant peptide of CENP-F antigen improved the ability to distinguish HCC from the healthy controls, with the AUC (95\% CI), sensitivity, specificity of 0.875 (95\% CI, 0.835-0.914), 75.2 and 84.9\%, respectively, better than AFP solely (Table IV). Meanwhile, improved diagnostic performance for detection of early-stage HCC was also observed for the combination, with AUC of 0.84, higher than AFP (0.72) solely (Table IV).

\section{Discussion}

Autoantibody to CENP-F has been recognized as a potential serological biomarker for the early diagnosis of HCC (22). As CENP-F is a high molecular weight protein of 3210 a.a, the immunogenicity of CENP-F antigen is critical for the sensitivity and specificity of detection of autoantibody to CENP-F. In the present study, we screened the predominant epitopes within 
Table III. Diagnostic value of 121-220 a.a and 1670-1791 a.a dominant peptides of CENP-F evaluated by ELISA analysis of samples of validation group.

\begin{tabular}{|c|c|c|c|c|c|}
\hline Dominant peptides & Cases & AUC value & $95 \% \mathrm{CI}$ & Sensitivity (\%) & Specificity (\%) \\
\hline & HCC vs. HC & & & & \\
\hline 121-220 a.a & & 0.749 & $0.692-0.807$ & 68.0 & 72.2 \\
\hline \multirow[t]{2}{*}{ 1670-1791 a.a } & & 0.628 & $0.563-0.693$ & 62.1 & 61.9 \\
\hline & HCC vs. LC & & & & \\
\hline $121-220$ a.a & & 0.559 & $0.491-0.627$ & 51.0 & 61.9 \\
\hline \multirow[t]{2}{*}{ 1670-1791 a.a } & & 0.541 & $0.474-0.609$ & 68.0 & 43.7 \\
\hline & Early-stage HCC vs. HC & & & & \\
\hline 121-220 a.a & & 0.743 & $0.674-0.812$ & 68.6 & 72.2 \\
\hline \multirow[t]{2}{*}{ 1670-1791 a.a } & & 0.584 & $0.500-0.668$ & 72.9 & 50.0 \\
\hline & Early-stage HCC vs. LC & & & & \\
\hline 121-220 a.a & & 0.465 & $0.383-0.546$ & 92.9 & 15.1 \\
\hline 1670-1791 a.a & & 0.518 & $0.435-0.602$ & 75.7 & 38.1 \\
\hline
\end{tabular}

a.a, amino acid; CENP-F, centromere protein F; ELISA, enzyme-linked immunosorbent assay; HCC, hepatocellular carcinoma; LC, liver cirrhosis; HC, healthy controls; AUC, area under curve; CI, confidence interval.
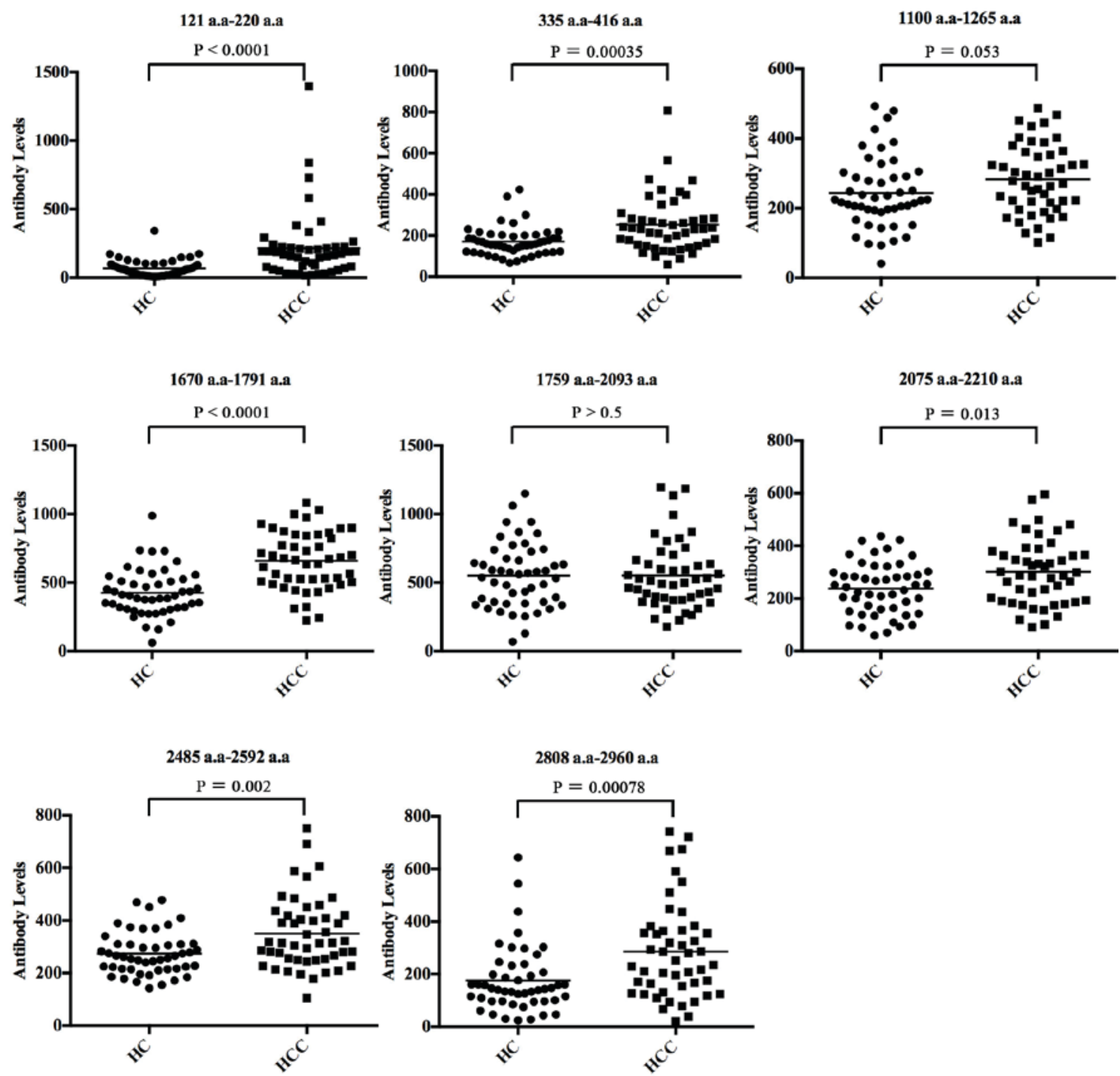

Figure 3. Scatter plot of the eight antigen peptides of CENP-F for discrimination between patients with hepatocellular carcinoma and healthy controls. Black horizontal lines indicate means. a.a, amino acid; CENP-F, centromere protein F. 
Table IV. Diagnostic value of the combination of AFP and 121-220 a.a or 1670-1791 a.a dominant peptides of CENP-F antigen.

\begin{tabular}{|c|c|c|c|c|c|}
\hline Dominant peptides & Cases & AUC value & $95 \% \mathrm{CI}$ & Sensitivity (\%) & Specificity (\%) \\
\hline & HCC vs. HC & & & & \\
\hline 121-220 a.a + AFP & & 0.875 & $0.835-0.914$ & 75.2 & 84.9 \\
\hline 1670-1791 a.a + AFP & & 0.827 & $0.779-0.876$ & 63.4 & 93.7 \\
\hline \multirow[t]{2}{*}{ AFP } & & 0.768 & $0.712-0.824$ & 53.6 & 100.0 \\
\hline & HCC vs. LC & & & & \\
\hline 121-220 a.a + AFP & & 0.702 & $0.641-0.763$ & 53.6 & 82.5 \\
\hline 1670-1791 a.a + AFP & & 0.700 & $0.638-0.761$ & 55.6 & 82.5 \\
\hline \multirow[t]{2}{*}{ AFP } & & 0.681 & $0.618-0.744$ & 53.6 & 82.5 \\
\hline & Early-stage HCC vs. HC & & & & \\
\hline 121-220 a.a + AFP & & 0.840 & $0.781-0.899$ & 81.4 & 72.2 \\
\hline 1670-1791 a.a + AFP & & 0.779 & $0.706-0.852$ & 51.4 & 93.7 \\
\hline \multirow[t]{2}{*}{ AFP } & & 0.721 & $0.639-0.804$ & 44.3 & 100.0 \\
\hline & Early-stage HCC vs. LC & & & & \\
\hline 121-220 a.a + AFP & & 0.626 & $0.543-0.709$ & 42.9 & 84.9 \\
\hline 1670-1791 a.a + AFP & & 0.639 & $0.557-0.721$ & 48.6 & 80.2 \\
\hline AFP & & 0.634 & $0.383-0.546$ & 44.3 & 82.5 \\
\hline
\end{tabular}

AFP, $\alpha$-fetoprotein; a.a, amino acid; CENP-f, centromere protein F; AUC, area under curve; CI, confidence interval; HCC, hepatocellular carcinoma; LC, liver cirrhosis; HC, healthy controls.
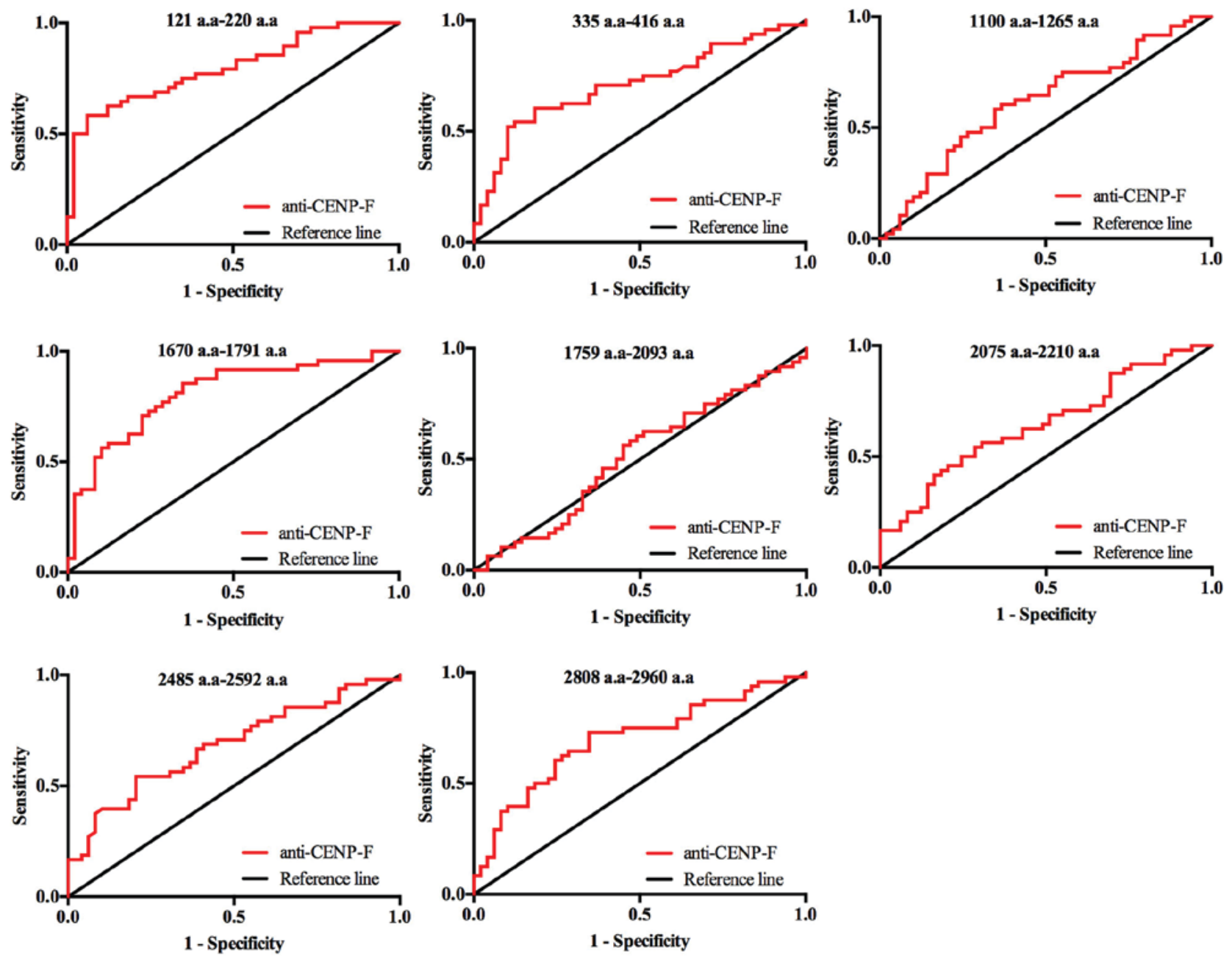

Figure 4. ROC curves of the eight individual CENP-F antigens for discriminating between patients with hepatocellular carcinoma and healthy controls. ROC, receiver operating characteristic; a.a, amino acid; CENP-F, centromere protein F. 
the full-length protein by bioinformatics analysis followed by ELISA detection, and selected two peptides (121-220 a.a and 1670-1791 a.a) for further clinical evaluation in a large cohort of HCC cases. Among the eight peptides of CENP-F tested, peptide 121-220 a.a demonstrated the best diagnostic value.

The CENP-F protein contains several motifs including tandem repeats that are sufficient for centromere, cytoplasm, or nuclear localization and for self-association (21) (Fig. 1B). Rattner et al (23) reported that the C-terminal end of CENP-F is especially antigenic; however, the exact regions were not determined. In other studies, Welner et al (24) evaluated the 1882-2153 a.a peptide of CENP-F antigen by indirect ELISA using overlapping 20-mer peptides of CENP-F spanning the amino acid sequence from 1882 to 2153 and two independent monoclonal antibodies to CENP-F in serum samples and found several peptides with potentially good immunogenicity. They further showed that approximately $50 \%$ of patients who were clinically tested for antinuclear antibody (ANA) and expressed antibodies to CENP-F were diagnosed with various kinds of cancer, confirming that such antibodies may function as circulating tumor markers.

In the present study we first predicted antigen epitope peptides and screened dominant epitopes through bioinformatics analysis, identifying eight candidate epitopes of CENP-F peptides. In subsequent evaluation of the serological responses of these eight antigens by indirect ELISA, two of the candidate epitopes showed better diagnostic value for HCC; the 121-220 a.a peptide of CENP-F had good specificity whereas the 1670-1791 a.a peptide had better sensitivity (Table III) suggesting that combined use of both peptides of CENP-F in further studies would enhance both the sensitivity and the specificity. The $121-220$ a.a peptide is located in the N-terminal of the CENP-F protein, which is quite different from the antigen reported previously, whereas the $1670-1791$ a.a peptide is located close to the 1882-2153 a.a peptide of CENP-F reported in other studies $(23,24)$. Finally, we conducted clinical evaluation in a large cohort of cases to validate the diagnostic value of the two candidate epitopes. Our results confirmed the promising diagnostic value of the 121-220 a.a peptide of CENP-F in the detection of early HCC (Table III); however, the 1670-1791 a.a peptide had lower diagnostic performance in the validation group compared with the screening group suggesting that further study with more cases is essential to understand the diagnostic value of this peptide of CENP-F.

CENP-F has already been reported as a potential biomarker for early-stage HCC $(15,22)$. Through high-throughput microarray analysis in large-scale cohorts of HCC and early-stage HCC cases, our previous study confirmed the diagnostic performance of anti-CENP-F in the detection of early HCC. In the present study, we evaluated the clinical significance of the 121-220 a.a peptide of CENP-F by indirect ELISA in 405 serum samples including patients with early-stage HCC, advanced HCC, and LC. The results showed that anti-CENP-F antibody had promising diagnostic performance in the detection of HCC and, moreover, could complement AFP leading to improved diagnosis of HCC or early HCC. However, the results also revealed the limited value of anti-CENP-F antibody in the discrimination of HCC and LC, consistent with our previous studies (22).
According to the ROC curve, we defined cases with antibody level of more than $125 \mathrm{ng} / \mathrm{ml}$ as positive for auto-antibody to CENP-F (121-220 a.a). The results showed that 48 of $70 \mathrm{HCC}$ cases $(68.6 \%)$ with TNM stage I, and 56 of 83 HCC cases (67.5\%) with TNM stage II or III, are positive for auto-antibody to $\mathrm{CENP}-\mathrm{F}(\mathrm{P}=0.512)$, suggesting CENP-F auto-antibody level is not related with the stage of HCC. However, the CENP-F had the highest prevalence of autoantibody positivity in HCC cases with TNM stage I implies that auto-antibody to CENP-F may have value in detection of early HCC.

There are some other limitations in our study. Although two candidate epitope peptides of CENP-F were identified, the exact structure of the antigen and the underlining mechanism remain unknown. In addition, the numbers of early-stage HCC cases were still limited, and further studies with a larger sample sizes would further warrant the findings in our study. Finally, as the pathology analysis of liver biopsy is the golden standard for diagnosis of $\mathrm{HCC}$, but in the present study only a part of HCC cases had pathology results. In our future study, we will use more pathologically confirmed HCC cases to further evaluate the diagnostic value of the CENP-F antibody.

In conclusion, through bioinformatics analysis and clinical evaluation, we identified the 121-220 a.a peptide as the peptide with highest immunogenicity in the CENP-F antigen. It also showed promising diagnostic value in detecting early-stage HCC and could therefore be a complement to AFP in early diagnosis of HCC.

\section{Acknowledgements}

This study was supported by grants from the National Natural Science Foundation of China (grant nos. 81071973 and 81602032), and Capital Foundation of Medical Developments (2016-2-2025) and State Key Projects Specialized on Infectious Diseases (2017ZX10201201-007-002).

\section{References}

1. Venook AP, Papandreou C, Furuse J and de Guevara LL: The incidence and epidemiology of hepatocellular carcinoma: A global and regional perspective. Oncologist 15 (Suppl 4): S5-S13, 2010.

2. Siegel R, Naishadham D and Jemal A: Cancer statistics, 2013. CA Cancer J Clin 63: 11-30, 2013.

3. Colli A, Fraquelli M, Casazza G, Massironi S, Colucci A, Conte D and Duca P: Accuracy of ultrasonography, spiral CT, magnetic resonance and alpha-fetoprotein in diagnosing hepatocellular carcinoma: A systematic review. Am J Gastroenterol 101: 513-523, 2006.

4. Poon D, Anderson BO, Chen LT, Tanaka K, Lau WY, Van Cutsem E, Singh H, Chow WC, Ooi LL, Chow P, et al: Management of hepatocellular carcinoma in Asia: Consensus statement from the Asian oncology summit 2009. Lancet Oncol 10: 1111-1118, 2009.

5. Marrero JA, Feng Z, Wang Y, Nguyen MH, Befeler AS, Roberts LR, Reddy KR, Harnois D, Llovet JM, Normolle D, et al: Alpha-fetoprotein, des-gamma carboxyprothrombin, and lectin-bound alpha-fetoprotein in early hepatocellular carcinoma. Gastroenterology 137: 110-118, 2009.

6. Zhang Z,Zhang Y, Wang Y,Xu Land Xu W: Alpha-fetoprotein-L3 and Golgi protein 73 may serve as candidate biomarkers for diagnosing alpha-fetoprotein-negative hepatocellular carcinoma. Onco Targets Ther 9: 123-129, 2016.

7. Ji J, Wang H, Li Y, Zheng L, Yin Y, Zou Z, Zhou F, Zhou W, Shen F and Gao C: Diagnostic evaluation of Des-gamma-carboxy prothrombin versus $\alpha$-fetoprotein for hepatitis B virus-related hepatocellular carcinoma in China: A large-scale, multicentre study. PLoS One 11: e0153227, 2016. 
8. Zhang J, Shao C, Zhou Q, Zhu Y, Zhu J and Tu C: Diagnostic accuracy of serum squamous cell carcinoma antigen and squamous cell carcinoma antigen-immunoglobulin $\mathbf{M}$ for hepatocellular carcinoma: A meta-analysis. Mol Clin Oncol 3: $1165-1171,2015$.

9. Shen Q, Fan J, Yang XR, Tan Y, Zhao W, Xu Y, Wang N, Niu Y, Wu Z, Zhou J, et al: Serum DKK1 as a protein biomarker for the diagnosis of hepatocellular carcinoma: A large-scale, multicentre study. Lancet Oncol 13: 817-826, 2012.

10. Lim TS, Kim DY, Han KH, Kim HS, Shin SH, Jung KS, Kim BK, Kim SU, Park JY and Ahn SH: Combined use of AFP, PIVKA-II and AFP-L3 as tumor markers enhances diagnostic accuracy for hepatocellular carcinoma in cirrhotic patients. Scand J Gastroenterol 51: 344-353, 2016.

11. Yu J, Wang ZJ, Chen LH and Dong WZ: Diagnostic value of serum squamous cell carcinoma antigen for hepatocellular carcinoma: A systematic review and meta-analysis. Scand J Clin Lab Invest 77: 8-14, 2017.

12. Giannelli G, Fransvea E, Trerotoli P, Beaugrand M, Marinosci F, Lupo L, Nkontchou G, Dentico P and Antonaci S: Clinical validation of combined serological biomarkers for improved hepatocellular carcinoma diagnosis in 961 patients. Clin Chim Acta 383: 147-152, 2007.

13. Marrero JA, Romano PR, Nikolaeva O, Steel L, Mehta A, Fimmel CJ, Comunale MA, D'Amelio A, Lok AS and Block TM: GP73, a resident Golgi glycoprotein, is a novel serum marker for hepatocellular carcinoma. J Hepatol 43: 1007-1012, 2005.

14. Hong Y: Autoantibodies against tumor-associated antigens for detection of hepatocellular carcinoma. World J Hepatol 7: $1581-1585,2015$.

15. Zhang JY, Zhu W, Imai H, Kiyosawa K, Chan EK and Tan EM: De-novo humoral immune responses to cancer-associated autoantigens during transition from chronic liver disease to hepatocellular carcinoma. Clin Exp Immunol 125: 3-9, 2001.
16. Dai Y, Liu L, Zeng T, Zhu YH, Li J, Chen L, Li Y, Yuan YF, Ma S and Guan XY: Characterization of the oncogenic function of centromere protein $\mathrm{F}$ in hepatocellular carcinoma. Biochem Biophys Res Commun 436: 711-718, 2013.

17. Zhu X, Mancini MA, Chang KH, Liu CY, Chen CF, Shan B, Jones D, Yang-Feng TL and Lee WH: Characterization of a novel 350-kilodalton nuclear phosphoprotein that is specifically involved in mitotic-phase progression. Mol Cell Biol 15: 5017-5029, 1995.

18. Ma L, Zhao X and Zhu X: Mitosin/CENP-F in mitosis, transcriptional control and differentiation. J Biomed Sci 13: 205-213, 2006.

19. Varis A, Salmela AL and Kallio MJ: Cenp-F (mitosin) is more than a mitotic marker. Chromosoma 115: 288-295, 2006.

20. Zhu X, Ding L and Pei G: Carboxyl terminus of mitosin is sufficient to confer spindle pole localization. J Cell Biochem 66: 441-449, 1997.

21. The national center for biotechnology information database. http://www/uniprot.org/uniprot/P49454.

22. Hong Y, Long J, Li H, Chen S, Liu Q, Zhang B, He X, Wang Y, $\mathrm{Li} \mathrm{H}, \mathrm{Li} \mathrm{Y}$, et al: An analysis of immunoreactive signatures in early stage hepatocellular carcinoma. EBioMedicine 2: 438-446, 2015.

23. Rattner JB, Rees J, Whitehead CM, Casiano CA, Tan EM, Humbel RL, Conrad K and Fritzler MJ: High frequency of neoplasia in patients with autoantibodies to centromere protein CENP-F. Clin Invest Med 20: 308-319, 1997.

24. Welner S, Trier NH, Houen G and Hansen PR: Identification and mapping of a linear epitope of centromere protein $\mathrm{F}$ using monoclonal antibodies. J Pept Sci 19: 95-101, 2013. 